\title{
Crowd Sourcing-based Deduplication in Big Data Environment
}

\author{
Bosco Nirmala Priya, D. Gayathri Devi
}

\begin{abstract}
Frequently, in reality, substances have at least two portrayals in databases. Copy records don't share a typical key as well as they contain mistakes that make copy coordinating a troublesome assignment. Mistakes are presented as the consequence of interpretation blunders, inadequate data, absence of standard configurations, or any mix of these components. In big data storage data is excessively enormous and productively store data is troublesome errand. To take care of this issue Hadoop instrument gives HDFS that oversees data by keep up duplication of data however this expanded duplication. In our anticipated strategy bigdata stream is given to the fixed size chunking calculation to make fixed size chunks. In this manuscript, we introduce an exhaustive investigation of the writing on crowd sourcing based big data deduplication technique. In our strategy is to create the guide diminish result after that MapReduce model is connected to discover whether hash esteems and are copy or not. To be familiar with the copy hash esteems MapReduce model contrasted these hash esteems and as of now put away hash esteems in Big data storage space. On the off chance that these hash esteems are now there in the Big data storage space, at that point these can be distinguished as copy. On the off chance that the hash esteems are copied, at that point don't store the data into the Hadoop Distributed File System (HDFS) else then store the data into the HDFS. we additionally spread various deduplication systems in crowd sourcing data's.
\end{abstract}

Keywords: Crowd-sourcing, deduplication, MapReduce, HDFS

\section{INTRODUCTION}

With the appearance of big data, data quality administration has turned out to could easily compare to ever. Normally, volume, speed and assortment are utilized to portray the key properties of big data [2].

Presently days expanding request of putting away an enormous sum data in computerized structure is calm testing task. In Big data storage, enormous measure of copy data is available. In huge organizations or big organizations enormous measure of data is prepared inside seconds.

Manuscript published on November 30, 2019.

* Correspondence Author

Ms. Bosco Nirmala Priya*, PhD. Research Scholar, Department of Computer Science, Sri Ramakrishna College of Arts and Science for Women, Coimbatore, Tamilnadu, India.

Dr. D. Gayathri Devi, Department of Computer Science, Sri Ramakrishna College of Arts and Science for Women, Coimbatore, Tamilnadu, India.

(C) The Authors. Published by Blue Eyes Intelligence Engineering and Sciences Publication (BEIESP). This is an open access article under the CC-BY-NC-ND license http://creativecommons.org/licenses/by-nc-nd/4.0/
This huge measure of data might be in the unstructured structure with no arrangement or media. This unstructured data may contain copy data utilized at various occasions so to distinguish copy data and make unstructured data into organized data configuration is a difficult undertaking [1].

Consistent with its significance, tending to the nearness of copy records in databases has been given proper consideration in the writing. Comparability methods dependent on characters, tokens, phonetic and numeric likenesses are utilized to match fields of data dependent on probabilistic, directed or semi-regulated based ways to deal with determine if a specific data record is a copy or not. These systems, while successful, have start-up expenses related with them to introduce with different look into tables dependent on which the copy discovery procedure will be completed [7].

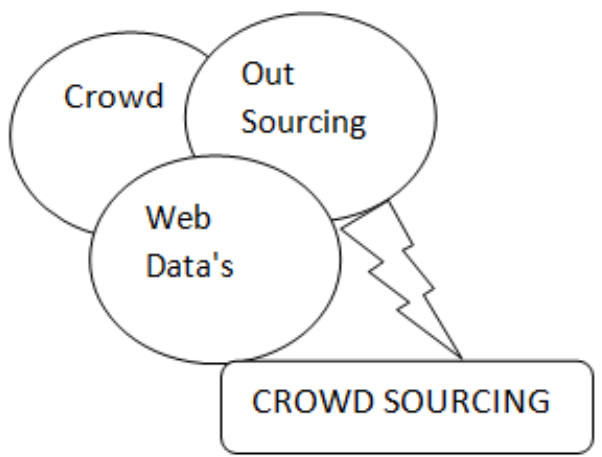

Figure 1: Crowd sourcing structure

In Figure 1 outlines, how the crowd sourcing data's are produced or recorded.

While from one perspective, it very well may be contended that such required expenses are common to have the suitable learning dataset dependent on which the procedure of copy records discovery will be semi-robotized; then again in the present focused occasions it additionally raises associations' issues identified with protection and security. Data is currently viewed as a pivotal business resource and the start-up procedure, by and large, requires a proficient data investigator who is outer to the association. In situations where the association manages delicate data this prompts protection and security issues with an inclination to keep this procedure in-house and secure [8]. Late propels in human figuring, to be specific crowd sourcing, has opened approaches to address the issue of deduplication and furthermore keep this procedure in-house. Human registering goes back to 1950 when Turing expressed that computerized PCs expect to achieve errands which should be possible by a human [9].

Published By:

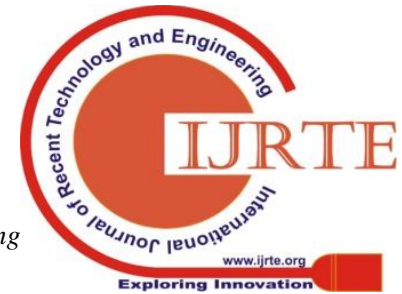


Rather than putting away various duplicates of a similar genomics data, our proposed deduplication framework will store just the data that is diverse alongside a guide to reproduce all data documents.

In this paper, we center around the issue of lexical heterogeneity and review different systems which have been created for tending to this issue. We center around the situation where the information is a lot of organized and appropriately divided records, i.e., we center essentially around instances of database records. Subsequently, we don't cover answers for different issues, for example, that of mirror discovery, wherein the objective is to distinguish comparable or indistinguishable Web pages (e.g., see [4], [5]). Additionally, we don't cover answers for issues, for example, anaphora goals [6] in which the issue is to find various notices of a similar element in free message (e.g., that the expression "Leader of the US" alludes to a similar element as "George W. Bramble"). We should take note of that the calculations produced for mirror discovery or for anaphora goals are frequently relevant for the assignment of copy identification. Strategies for mirror location have been utilized for discovery of copy database records and procedures for anaphora goals are usually utilized as a basic piece of deduplication in relations that are removed from free content utilizing data extraction frameworks [6].

\section{BACKGROUND STUDY}

Kumar, N., Rawat, R., \& Jain, S. C. [1] presents a container based system. In proposed procedure various basins are utilized to store data and when same data is gotten to by guide lessen for example as of now put away in container then that data will be disposed of so this strategy unquestionably builds productivity of bigdata storage. Results demonstrates that in proposed instrument deduplication proportion is high, data size decrease is high hash time and chunk time is low as contrast with existing fixed size chunking strategy.

Abboura, A., Sahrl, S., Ouziri, M., \& Benbernou, S. [2] presented CrowdMD which is a MDs age framework. it has a mixture (crowdsourcing-algorithmic) engineering that permits to produce this sort of standards over a lot of data. Our difficult issue was to guarantee a decent bargain between the expense and nature of results.

Huang, Z., Li, H., Li, X., \& He, W. [3] propose another stateful data directing calculation, SS-Dedup, for bunch based deduplication framework. SS-Dedup uses of data comparability dependent on Border's Theorem. The principle commitment of our work is that SS-Dedup keeps up LRU reserves in customer server in memory to store the recorded fingerprints data for every datum servers, not the same as other stateful calculations just apply stores in data server to decrease unique mark query times. Thus, customers can choose the objective data server of new super-chunk legitimately for some situation. As such, SSDedup improves framework throughput further as well as decreases pointless correspondence overhead.

Ma, K., Dong, F., \& Yang, B. [4] presented a huge scale blueprint free data deduplication approach in parallel. This methodology uses the MapReduce system with versatile sliding window. We have told the best way to change Sort-
Map-Reduce way to deal with an effectively parallelized deduplication approach with MapReduce in an assessment utilizing diagram free rearing record stores. We proposed three MapReduce-based executions: Sort-Map-Reduce, Partition-Sort-Map-Reduce and Partition-Sort-Map-Reduce with Adaptive Sliding Window. The proposed multi-pass coordinated arrangement is more productive has a straightforward execution of rehashed single Partition-SortMap-Reduce with Adaptive Sliding Window.

Daren C Brabham [6] given a prologue to crowd-sourcing through definitions built up by its pioneers and delineated through an accumulation of case models. crowd-sourcing can be clarified through a hypothesis of crowd knowledge an activity of aggregate insight, yet we ought to stay disparaging of the model for what it may do to individuals and how it might reinstitute long-standing components of persecution through new talks.

Manas Joglekar, Hector Garcia-Molina, and Aditya Parameswaran [8] acquainted procedures with produce certainty interims for specialist blunder rate gauges, in this way empowering a superior assessment of laborer quality. We demonstrate that our methods create right certainty interims on a range of genuine world datasets, and exhibit wide appropriateness by utilizing them to oust ineffectively performing laborers, and give certainty interims on the exactness of the appropriate responses.

\section{BIG DATA DEDUPLICATION}

\section{A) DEDUPLICATION PROCESS}

Data deduplication (otherwise called substance goals, object coordinating and record linkage) is such a dataescalated and execution basic assignment that can profit by distributed computing and parallel registering. The contribution of deduplication procedure is called grimy data with such a large number of copies, and the yield of deduplication procedure is the division of deduplication from the info. deduplication is the initial step of data cleaning. Given at least one data sets, data deduplication is connected to decide all items alluding to the equivalent or comparable worth. Enthusiasm for deduplication research and practice gives off an impression of being becoming proportionate with the expansion in data volume and multifaceted nature.

\section{B) DEDUPLICATION TECHNIQUES}

As of late, data deduplication is a functioning and hot research theme with some parallel procedures. Targeting improving the deduplication quality, numerous methodologies have been proposed and assessed as depicted in late reviews. The time multifaceted nature is dissected as the evaluated number of examinations.

\section{i) Traditional blocking method}

This strategy has been utilized in deduplication since the 1960s . All records that have a similar blocking key are conveyed into a similar square, and just records inside a similar square are then contrasted and one another. 


\section{ii) Sorted neighborhood method}

Another well known blocking approach is SNM. A blocking key $\mathrm{k}$ is resolved for every one of $\mathrm{n}$ objects. For the most part, one quality or a couple of composite characteristics structure the blocking key. A short time later, the articles are arranged by these blocking keys. A sliding window with a fixed size $\omega$ is then moved over the arranged items inside a separation of $\omega-1$ for examination.

\section{iii) Canopy clustering method}

This deduplication technique depends on utilizing a computationally shabby bunching way to deal with make high dimensional covering groups, from which squares of applicant articles would then be able to be produced. Both of these measures to figure the similitude's depend on tokens. The key of this methodology is to make the covering groups.

\section{iv) Deduplication method in parallel}

A few present day parallel deduplication strategies have been proposed to improve the exhibition utilizing multicenters, pipelines, GPU and MapReduce methods. Targeting combining every one of the data identifying with the equivalent or comparable article in parallel, Febrl framework has depicted the probabilistic parallel coordinating to demonstrate how the match calculation can be parallelized among accessible centers on a solitary hub.

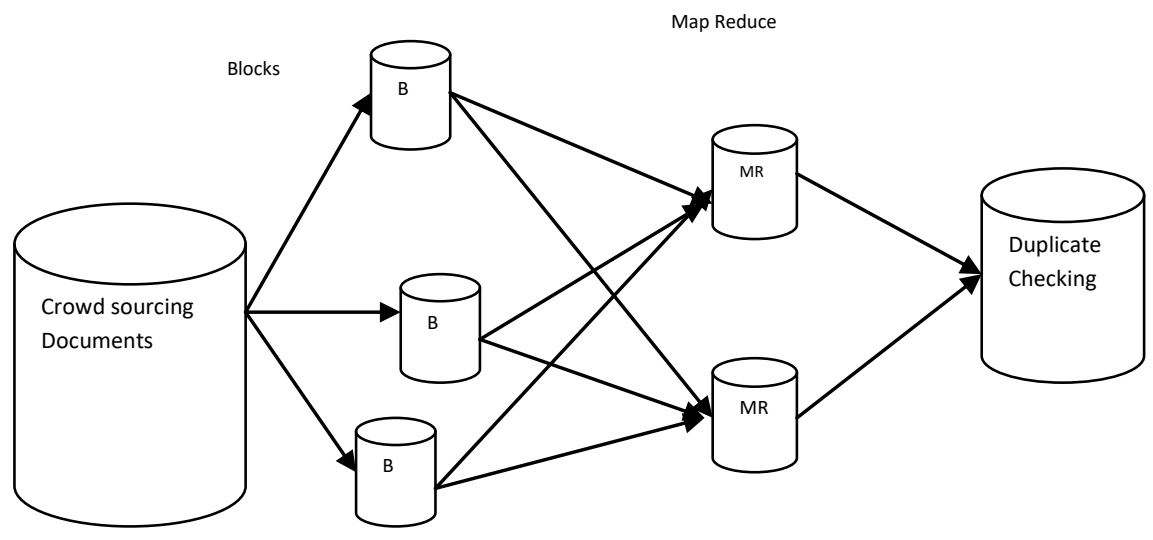

Fig 1: Deduplication with Map reduce using crowd-sourcing documents

\section{C) MAPREDUCE FRAMEWORK}

The MapReduce structure is embraced as the computational model when deduplication the rearing data for further data mining. A prevalent free usage is Apache Hadoop. The model is enlivened by the guide and decrease works usually utilized in practical programming, despite the fact that their motivation in the MapReduce system (see this figure 1) isn't equivalent to their unique structures. The procedure is isolated into two stages: map and decrease. Designers indicate Map capacities which take an info pair and creates a lot of halfway key/esteem sets.

\section{D) DEDUPLICATION WITH MAPREDUCE}

A few specialists have proposed the new acknowledgment of the general deduplication with MapReduce, which is moderately clear by actualizing hindering inside the guide work and by executing coordinating inside the diminish work. To this end, map initially decides the blocking key for each item. The MapReduce system gathering objects with a similar blocking key to squares and redistributes them. The diminish step at that point coordinates the articles inside one square (see this figure 1). The guide and lessen strategy is executed in parallel. The coordinating outcomes are disjoint by definition and can in this manner effectively be converged to acquire the total last outcomes.

\section{COMPARATIVE ANALYSIS OF SURVEY}

Normally the proposed sort is made by basically considering the various drawbacks of the present frameworks organization correspondence.

Table 1: Evaluation on various authors views.

\begin{tabular}{|l|l|l|c|}
\hline Paper Name & Methodology & Limitations & Pumber. \\
& & & \\
\end{tabular}




\begin{tabular}{|c|c|c|c|}
\hline $\begin{array}{l}\text { Bucket based data deduplication } \\
\text { technique for big data storage system by } \\
\text { Kumar, N., Rawat, R., \& Jain, S. C. }\end{array}$ & $\begin{array}{l}\text { proposed bucket based data } \\
\text { deduplication strategy is displayed and } \\
\text { bigdata stream is given to the fixed } \\
\text { size chunking calculation to make } \\
\text { fixed size chunks. }\end{array}$ & $\begin{array}{l}\text { In fixed size chunking there are fixed size } \\
\text { chunks are made yet when there is a few } \\
\text { changes in data at that point there might be an } \\
\text { issue limit move issue. }\end{array}$ & [1] \\
\hline $\begin{array}{l}\text { CrowdMD: Crowdsourcing-based } \\
\text { approach for deduplication by Abboura, } \\
\text { A., Sahrl, S., Ouziri, M., \& Benbernou, S. }\end{array}$ & $\begin{array}{l}\text { CrowdMD, a mixture machine- } \\
\text { crowd framework for producing } \\
\text { MDs(Matching dependencies). }\end{array}$ & $\begin{array}{l}\text { discovery of copies by applying MDs and } \\
\text { propose another technique to accommodate } \\
\text { their tangled qualities. }\end{array}$ & [2] \\
\hline $\begin{array}{l}\text { Large-Scale } \text { Schema-Free Data } \\
\text { Deduplication Approach with Adaptive } \\
\text { Sliding Window Using MapReduce by } \\
\text { Ma, K., Dong, F., \& Yang, B. }\end{array}$ & $\begin{array}{l}\text { new composition free data } \\
\text { deduplication approach in parallel in } \\
\text { the part of rearing data deduplication } \\
\text { identified with sanitation. }\end{array}$ & $\begin{array}{c}\text { parallel deduplication methods with } \\
\text { MapReduce. }\end{array}$ & [4] \\
\hline $\begin{array}{l}\text { GDedup: Distributed File System Level } \\
\text { Deduplication for Genomic Big Data by } \\
\text { Bartus, P., \& Arzuaga, E. }\end{array}$ & $\begin{array}{l}\text { offered GDedup, a distributed file } \\
\text { system level deduplication storage } \\
\text { system for genomic big data }\end{array}$ & $\begin{array}{l}\text { to get better data storage capability and } \\
\text { effectiveness in distributed file systems }\end{array}$ & [5] \\
\hline $\begin{array}{l}\text { Evaluating the Crowd with Confidence } \\
\text { by Manas Joglekar, Hector Garcia-Molina, } \\
\text { and Aditya Parameswaran, }\end{array}$ & $\begin{array}{l}\text { We offer a novel disagreement- } \\
\text { based method for estimating } \\
\text { worker excellence beside with } \\
\text { assurance intervals }\end{array}$ & $\begin{array}{ll}\bullet & \text { Varying Task Difficulty } \\
\text { - } & \text { Multiple Task Types } \\
\text { - } & \text { Non-binary tasks }\end{array}$ & [8] \\
\hline
\end{tabular}

We have compared for various authors proposed system V. RESULTS AND DISCUSSION accuracy.

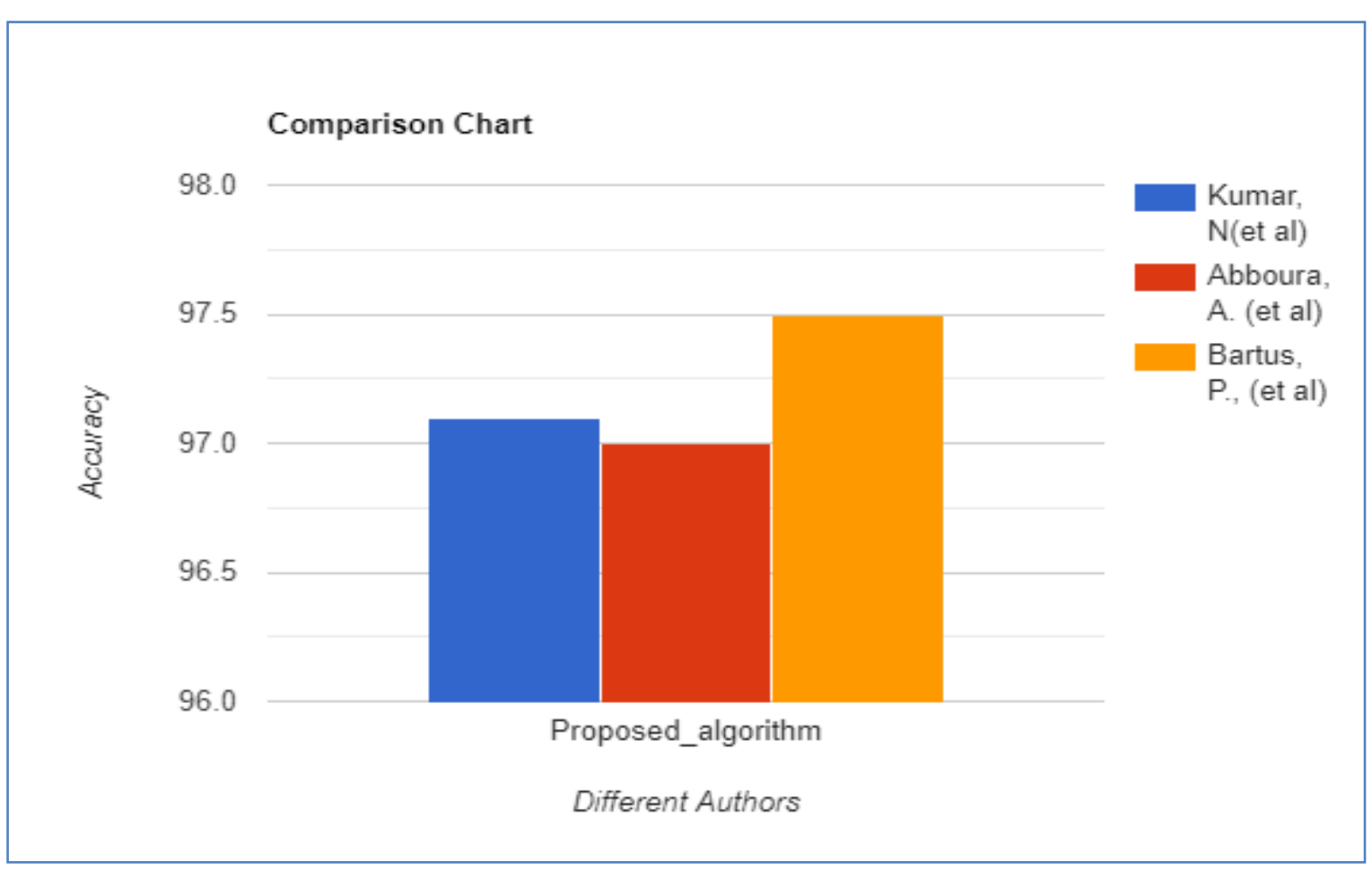

Fig 2: Comparison Chart

In Figure 2 represents the comparision between some of authors proposed systems and here we have given the proposed level accuracy is displayed.

Subsequent to making an example data set, we register the deduplication proportion and the level of copy chunks for each altered arrangement of records and the reference

set. Let chunk Size signify the chunk size, DedupRatio indicate the deduplication proportion, and DupCh mean the level of copy chunks. The deduplication proportion is characterized as the proportion between the first data size and the data size in the wake of dispensing with the copies.

A higher DedupRatio demonstrates a high excess in the document content while a lower proportion demonstrates a high number of one of a kind chunks in the record.

Blue Eyes Intelligence Engineering \& Sciences Publication 
So also, a high DupCh demonstrates that the record substance can be spoken to by a blend of a little subset of interesting chunks, while a low worth shows profoundly one of a kind chunk content. We utilized the accompanying chunk Size values: 512 B, $1 \mathrm{~KB}, 2 \mathrm{~KB}$, and $4 \mathrm{~KB}$. To process the level of copy chunks and the deduplication proportion, the chunk identifiers from the first arrangement of documents were contrasted and the chunk identifiers from each changed arrangement of records.

\begin{tabular}{|l|l|l|l|l|l|l|l|l|l|}
\hline $\mathrm{A}$ & $\mathrm{A}$ & $\mathrm{B}$ & $\mathrm{C}$ & $\mathrm{D}$ & $\mathrm{E}$ & $\mathrm{F}$ & $\mathrm{G}$ & $\mathrm{H}$ & $\mathrm{I}$ \\
\hline
\end{tabular}

\begin{tabular}{|r|r|r|r|r|r|r|r|r|r|}
\hline A & A & B & X & D & E & Y & G & H & I \\
\hline
\end{tabular}

Figure 3: Each file contains 10 chunks of the same size.

Chunks $\mathrm{C}$ and $\mathrm{F}$ are supplanted by chunks $\mathrm{X}$ and $\mathrm{Y}$, separately. Chunks A, B, D, E, G, H, and I are copies. We demonstrate a case of these measurements utilizing Figure 3, which demonstrates the subsequent chunks from two records after deduplication. In this hypothetical model, we expected that after deduplication, each record was isolated into 10 chunks of a similar size.

The first is the reference record, and the subsequent one is the adjusted document. During deduplication, the chunk identifier together with a counter are recorded into the genomics deduplication database. The all out number of chunks, the quantity of extraordinary chunks (count=1), and the quantity of particular chunks were registered by issuing questions to the database. The level of copy chunks is:

DupCh $=\frac{\text { Total chunks }- \text { Unique chunks }}{\text { Total chunks }} X(100 \%)$

The deduplication ratio is given by the following formula:

DedupRatio $=\frac{\text { Total chunks }}{\text { Distinct chunks }}$

In this model, the absolute number of chunks is 20, there are 11 unmistakable chunks, and 4 interesting chunks. The level of copy chunks and the deduplication proportion are:

DupCh $=\frac{20-4}{20} X(100 \%)=80 \%$

DedupRatio $=\frac{20}{11}=1.82$

The memory asset prerequisites are moderately little, just one record must be prepared. Deduplication needs all the more handling force.

\section{CONCLUSION}

In this review, we have introduced a complete study of the current procedures utilized for discovering deduplication in big data condition with crowd sourcing. As database frameworks are ending up increasingly typical, data cleaning will be the foundation for redressing blunders in frameworks which are amassing huge measures of mistakes once a day. In spite of the expansiveness and profundity of the exhibited strategies, we accept that there is still space for considerable improvement in the present best in class. The absence of institutionalized, huge scale benchmarking data sets can be a big hindrance for the further improvement of the field as it is practically difficult to convincingly contrast new procedures and existing ones. The majority of the copy location frameworks accessible today offer different algorithmic methodologies for accelerating the copy identification process. The changing idea of the copy recognition process additionally requires versatile strategies that identify various examples for copy location and consequently adjust after some time. For instance, a foundation procedure could screen the present data, approaching data, and any data sources that should be consolidated or coordinated, and choose, in light of the watched blunders, regardless of whether a modification of the copy recognition procedure is fundamental or not. Another related part of this test is to create techniques that license the client to infer the extents of blunders expected in data cleaning ventures.

\section{REFERENCES}

1. Kumar, N., Rawat, R., \& Jain, S. C, "Bucket based data deduplication technique for big data storage system". 2016 5th International Conference on Reliability, Infocom Technologies and Optimization (Trends and Future Directions) (ICRITO).

2. Abboura, A., Sahrl, S., Ouziri, M., \& Benbernou, S., "CrowdMD: Crowdsourcing-based approach for deduplication". 2015 IEEE International Conference on Big Data (Big Data).

3. Huang, Z., Li, H., Li, X., \& He, W, "SS-dedup: A high throughput stateful data routing algorithm for cluster deduplication system". 2016 IEEE International Conference on Big Data (Big Data).

4. Ma, K., Dong, F., \& Yang, B., "Large-Scale Schema-Free Data Deduplication Approach with Adaptive Sliding Window Using MapReduce". The Computer Journal, 58(11), 3187-3201.

5. Bartus, P., \& Arzuaga, E., "GDedup: Distributed File System Level Deduplication for Genomic Big Data", 2018 IEEE International Congress on Big Data (BigData Congress).

6. Daren C Brabham, "Crowdsourcing as a model for problem solving an introduction and cases," Convergence: the international journal of research into new media technologies, vol. 14, no. 1, pp. 75-90, 2008.

7. Ahmed K Elmagarmid, Panagiotis G Ipeirotis, and Vassilios S Verykios, "Duplicate record detection: A survey," IEEE Transactions on knowledge and data engineering, vol. 19, no. 1, pp. 1-16, 2007.

8. Manas Joglekar, Hector Garcia-Molina, and Aditya Parameswaran, "Evaluating the crowd with confidence," presented at the Proceedings of the 19th ACM SIGKDD international conference on Knowledge discovery and data mining, Chicago, Illinois, USA, 2013

9. Kumar, A., Vengatesan, K., Vincent, R., Rajesh, M., Singhal, A. : A novel Arp approach for cloud resource management; International Journal of Recent Technology and Engineering (IJRTE) at Volume-7 Issue-6, March 2019

10. T. Narmadha, J. Gowrishankar, M. Ramkumar, and K. Vengatesan,"Cloud Data Center Based Dynamic Optimizing Replica Migration",J. Comput. Theor. Nanosci. 16, 576-579

11. Kesavan, S., Saravana Kumar, E., Kumar, A., Vengatesan, K. : An investigation on adaptive HTTP media streaming Quality-ofExperience (QoE) and agility using cloud media services; International Journal of Computers and Applications

12. M. Allahbakhsh, B. Benatallah, A. Ignjatovic, H. R. MotahariNezhad, E. Bertino, and S. Dustdar, "Quality Control in Crowdsourcing Systems: Issues and Directions," IEEE Internet Computing, vol. 17, no. 2, pp. 76-81, 2013. 